\title{
Inferiority, Not Similarity of the Decoy to Target, Is What Drives the Transfer of Attention Underlying the Attraction Effect: Evidence from an Eye-Tracking Study with Real Choices
}

DOI:

10.1037/npe0000104

Document Version

Accepted author manuscript

Link to publication record in Manchester Research Explorer

\section{Citation for published version (APA):}

Krol, M., \& Krol, M. (2019). Inferiority, Not Similarity of the Decoy to Target, Is What Drives the Transfer of Attention Underlying the Attraction Effect: Evidence from an Eye-Tracking Study with Real Choices. Journal of Neuroscience, Psychology, and Economics. https://doi.org/10.1037/npe0000104

\section{Published in:}

Journal of Neuroscience, Psychology, and Economics

\section{Citing this paper}

Please note that where the full-text provided on Manchester Research Explorer is the Author Accepted Manuscript or Proof version this may differ from the final Published version. If citing, it is advised that you check and use the publisher's definitive version.

\section{General rights}

Copyright and moral rights for the publications made accessible in the Research Explorer are retained by the authors and/or other copyright owners and it is a condition of accessing publications that users recognise and abide by the legal requirements associated with these rights.

\section{Takedown policy}

If you believe that this document breaches copyright please refer to the University of Manchester's Takedown Procedures [http://man.ac.uk/04Y6Bo] or contact uml.scholarlycommunications@manchester.ac.uk providing relevant details, so we can investigate your claim.

\section{OPEN ACCESS}


1 Inferiority, Not Similarity of the Decoy to Target, Is What Drives the Transfer of Attention

2 Underlying the Attraction Effect: Evidence from an Eye-Tracking Study with Real Choices 3

7 michal.krol@manchester.ac.uk (corresponding author)

8 Dr. Magdalena Krol

9 University of Social Sciences and Humanities, Aleksandra Ostrowskiego 30b, 50-001 Wrocław,

10 Poland, mkrol1@swps.edu.pl

11

12

13 Acknowledgment: This work was supported by the Polish Ministry of Science and Higher

14 Education grant number BST/WROC/2016/A/6.

15

16 


\section{Abstract}

19 Recent studies reported that the attraction effect, whereby inferior decoys cause choice reversals,

20 fails to replicate if the choice options are presented in a pictorial rather than abstract numerical

21 form. We argue that the pictorial setting makes the similarity between decoy and target salient,

22 while the abstract one emphasizes the inferiority relationship between them, crucial for the effect

23 to occur. Thus, we used a novel experimental design in which both similarity and inferiority are

24 equally easy to judge, their relative strength simple to manipulate, and choices incentivized

25 rather than hypothetical. Using eye-tracking, we found that both the transfer of attention towards

26 an undesirable target and choice reversal likelihood increase when the decoy is more strongly

27 inferior but less similar to the target. This suggests that a key mechanism in the attraction effect

28 is that, by virtue of its inferiority, a decoy projects a spotlight of attention towards the target,

29 making it more attractive.

30 KEYWORDS: eye-tracking; choice reversal; attraction effect 
One of the most prominent context effects in consumer choice is the attraction effect

33 (Huber, Payne, \& Puto, 1982). This occurs when choosing between different options, say A and

34 B, each of which is better than the other in some respects. Adding a third option, C ('decoy'),

35 that is in every respect worse than B ('target'), but still in some respects better than A

36 ('competitor'), makes people more likely to choose B, even if they would have preferred A to B

37 in the absence of $\mathrm{C}$.

A number of existing theories, backed by behavioral experiments, have pointed to

39 attention transfers as a key mechanism behind the attraction effect. For instance, Roe,

40 Busemeyer, and Townsend (2001) proposed a 'Multialternative Decision Field Theory', whereby

41 the switching of attention between choice option attributes determines the eventual choice. The

42 theory highlights the fact that, on the one hand, when the decoy is very similar to the target but

43 not clearly inferior, it can reduce the preference for the target via a 'similarity effect'. On the

44 other hand, when the decoy's inferiority is apparent, it can increase the attractiveness of a highly

45 similar target via lateral inhibition, drawing attention towards the attributes where it is superior

46 (see also Ariely \& Wallsten, 1995; Bhatia, 2013; Bordalo, Gennaioli, \& Shleifer, 2013). The

47 more recently developed 'Multiattribute Linear Ballistic Accumulator Model' (J. S. Trueblood,

48 Brown, \& Heathcote, 2014) also assumes an attentional mechanism, whereby the fact that the

49 decoy is closer to the target than to the competitor results in more attention being allocated to the

50 comparison between the difficult to discriminate decoy and target options, ultimately revealing

51 and highlighting the superiority of the target. Finally, Ok, Ortoleva, and Riella (2015) theorized

52 that, in a two-stage process, a decoy first acts as a 'spotlight', narrowing down a person's

53 attention to an 'attraction region', before choosing from within this region at stage two (also see

54 Lleras, Masatlioglu, Nakajima, \& Ozbay, 2017 for a related 'limited consideration' model). 
Despite terms like 'attention' or 'saliency' featuring prominently in these explanations,

56 and despite eye-tracking being often used to investigate other forms of decision bias (e.g., Król

$57 \&$ Król, in press), there has been little attempt to use it to study the visual attention patterns

58 accompanying decoy-induced preference reversals. Such an analysis seems fruitful, because

59 research on value-based choice (in the absence of decoys) demonstrated that attention guides

60 choices in a positive feedback loop, whereby a stronger preference for an option causes more

61 attention to be allocated to it, and vice versa (Ashby, Jekel, Dickert, \& Glöckner, 2016; Krajbich,

62 Armel, \& Rangel, 2010; Reutskaja, Nagel, Camerer, \& Rangel, 2011; Shimojo, Simion,

63 Shimojo, \& Scheier, 2003). Nevertheless, we are aware of only a very small number of process-

64 tracing studies of context effects, most of which were based on functional magnetic resonance

65 imaging (W. Hedgcock, Rao, \& Chen, 2009; Hu \& Yu, 2014; Li, Michael, Balaguer, Herce

66 Castañón, \& Summerfield, 2018; Mohr, Heekeren, \& Rieskamp, 2017), and only one of which

67 used eye-tracking. In this study, Noguchi and Stewart (2014) found that, in scenarios that induce

68 context effects, people compare alternatives in pairs, one attribute at a time. However, their

69 otherwise comprehensive design did not allow for an identification of which individual choices

70 were actually affected by decoys (that is, it is impossible to tell if a subject would choose

71 differently from a given competitor-target pair in the absence of a decoy).

72 To address the above problem, similarly to Hedgcock, Rao, and Chen (2016), we used a

73 repeated-measures design, in which subjects face each competitor-target combination both with

74 and without a decoy (separated by other decision trials as well as distractor tasks). However,

75 unlike any existing studies, we combined this type of a repeated-measures design with eye-

76 tracking. To illustrate the purpose of this setup, consider the two-attribute choice problem

77 depicted in Figure 1. Suppose that we have established that a given participant prefers option 'c' 
78 to ' $t$ ' in the absence of any other choice options. The question we ask in this study is: if we then

79 present the participant with the same choice, but with a decoy that is slightly inferior but very

80 similar to the target (' $\mathrm{d}_{1}$ ') would it result in a greater transfer of visual attention from the

81 competitor ('c') to the target (' $\mathrm{t}$ ') than if we instead used a decoy that is highly inferior but not

82 very similar to the target (' $\mathrm{d}_{2}$ ')?

83 [Figure 1 about here]

The above question is all the more important given that it can be linked to the recent

85 debate about the robustness of the attraction effect. In particular, the authors of two extensive

86 replication projects have argued that the effect is only reproduced if the choice options are

87 presented as combinations of abstract numerical attributes, but fails to occur under a more

88 realistic, pictorial representation (Frederick, Lee, \& Baskin, 2014; Yang \& Lynn, 2014). We

89 believe that the reason for the discrepancy is that the numerical representation makes the

90 inferiority relationship between the decoy and the target salient, as it is easy for subjects to notice

91 that one is worse than the other in terms of, for example, both the quality index and the price. In

92 contrast, displaying options as pictures typically emphasizes the similarity between decoy and

93 target - in most stimuli examples presented in the two replication studies it is immediately clear

94 that two of the three options may be perceived as a group, e.g. one gets to choose between two

95 very similarly packaged varieties of the same brand of popcorn and one visually distinct product

96 of another brand. At the same time, as noted by Simonson (2014), this makes it very hard for

97 subjects to tell which option in the pictorial replication studies is the dominated, inferior one,

98 whereas, according to Huber, Payne, and Puto (2014) 'attraction depends on the ability to

99 identify the dominance relationship quickly and unambiguously'. Even in those pictorial

100 replications in which inferiority is clear (e.g. displaying pictures of an orange, a fresh apple, and 
101 a spoiled apple), the problem might, in turn, be that undesirable options are known to attenuate 102 the attraction effect (Malkoc, Hedgcock, \& Hoeffler, 2013).

104 literature review, is that over $98 \%$ of studies of the attraction effect, whether pictorial or abstract, 105 are based on hypothetical choices, typically using MTurk and similar online surveys. Thus, the 106 results are potentially affected by subjects' lack of attention, motivation, and automatic response 107 patterns (Lichters, Bengart, Sarstedt, \& Vogt, 2017; Smith, Roster, Golden, \& Albaum, 2016). 108 With this in mind, we sought to investigate the role of similarity vs. inferiority in the 109 attraction effect via a design in which: a) both relationships are equally easy for the subject to 110 observe, i.e. equally salient; b) the extent to which options are inferior or similar to one another 111 is precisely measured and manipulated; and c) subjects receive whatever they select, i.e. choices 112 are non-hypothetical. In particular, the first objective (a) is achieved by presenting the choice 113 options in a spatial grid, i.e. as in Figure 1.

114 Based on the discussed abstract vs. pictorial discrepancy reported by recent studies, we 115 hypothesized that introducing a decoy inferior to an option one did not choose in decoy's

116 absence will transfer attention (measured by eye fixation duration) to the target from the 117 competitor (that is, a greater proportion of the fixation time will be allocated to an unattractive 118 target if a decoy dominated by that target is added to the choice set). However, this effect will be 119 weaker if the decoy is more similar but less inferior to the target (' $\mathrm{d}_{1}$ ' in Figure 1, as opposed to 120 ' $\mathrm{d}_{2}$ '). If confirmed, such a result would indicate that the attentional spotlight cast on the target by 121 virtue of its superiority rather than similarity to the decoy is strong enough to offset the natural 122 tendency to perceive spatially close/similar objects as a group and divert more attention towards 123 them (Kimchi, Yeshurun, Spehar, \& Pirkner, 2016). 
125 the possibility of predicting, based on attention to choice options in the presence of a decoy,

126 whether the decoy actually influenced the subject's preferences, i.e. whether or not the choice

127 would have been different if the decoy were absent. In particular, we expected that increased

128 attention to both the decoy and the competitor despite choosing the target might indicate a

129 'genuine' preference for the competitor that has been altered by the decoy. We discussed how 130 such an approach might be useful in the evaluation of real-world marketing strategies.

\section{Method}

\section{Subjects}

The experiment was carried out at a large private university, where a total of 68

135 undergraduate and postgraduate student subjects were recruited. Their average age was 21.88

$136(\mathrm{SD}=3.18), 39$ of them were female, and all had normal or corrected to normal eyesight.

\section{Stimuli and Design}

Subjects repeatedly chose (without time limit) one out of two or one out of three bundles

139 of two types of goods: (1) shopping vouchers redeemable at any store of a big supermarket

140 chain, including a store located in the vicinity of the experiment location; (2) food and drink

141 vouchers redeemable at the local university canteen. All monetary values stated below are in the

142 local currency, a unit of which is worth approximately 0.3 USD. For instance, in a given choice

143 trial a subject might be asked to choose one of the following two bundles: (1) a supermarket

144 voucher worth 4 and a canteen voucher worth 16; or (2) a supermarket voucher worth 14 and a 145 canteen voucher worth 8. 
An example of how the choice was presented to subjects is illustrated in Figure 2. The

147 setting was partly inspired by a typical retailer's taxonomical shelf design, where products that

148 are more similar are located closer together, but where it is equally simple to locate products that

149 are inferior in quality or price (e.g. basic, low-quality products are placed in the bottom shelves).

150 This is known to facilitate the consumers' evaluation of the products and increases their

151 satisfaction (Meier \& Robinson, 2004; Mogilner, Rudnick, \& Iyengar, 2008).

Accordingly, in our case, the subjects' assessment of both similarity and inferiority was

153 facilitated by presenting the voucher bundles on a spatial grid, where each 'disk' corresponds to

154 a single bundle. In the example in Figure 2, its X-axis position (or the number in the left half of

155 the disk) represents the value of the supermarket voucher included in the bundle, and the Y-axis

156 position is the value of the included canteen voucher. Thus, one bundle was inferior to another if

157 positioned to the bottom left of it, while similarity was simple to judge based on proximity.

As explained in the 'Payoffs' section, our subjects were motivated to reveal their true

159 preferences. As such, they were unlikely to be biased by the visual presentation of the choice

160 options, with the grid only there to support their assessment of the relationships between them

161 (as shown by existing research, visual decision support tools can help people during multi-

162 attribute choice, see e.g. Samek, Hur, Kim, \& Yi, 2016).

163 Nevertheless, to counterbalance potential artefacts associated with the relative positioning

164 of the choice options, for half of the subjects the canteen and supermarket voucher values were

165 instead indicated by the $\mathrm{X}$ and $\mathrm{Y}$ axis respectively (as expected, we found no difference between

166 the two groups of subjects). Furthermore, as detailed later, the relative positioning of the choice

167 options was included as an independent variable in the regression model (rather than held

168 constant across trials) allowing us to control and investigate its effect on attention and choices. 
[Figure 2 about here]

After every trial, subjects carried out a distractor task (Figure 3), i.e. needed to say if a

171 random math inequality is true (e.g. ' $16-4>3+11$ '), and were reminded which number in a

172 disk representing each bundle corresponds to which type of voucher (this was done via an

173 example bundle, randomly generated in each instance of the task to additionally obstruct any

174 attempts at memorizing the previous choice options). The purpose of the distractor task was to

175 avoid carryover memory/learning effects and psychological reactivity (Ahn, Kim, \& Ha, 2015;

176 Hutchinson, Kamakura, \& Lynch, 2000), by removing the primary task (choice-related)

177 information from short-term memory (Bjork \& Whitten, 1974).

178 [Figure 3 about here]

\section{Choice Trial Specification}

In each of the first 30 trials of the experiment, each subject would choose one of two

181 bundles. The composition of each pair of bundles was drawn randomly subject to a number of

182 constraints. That is, we considered all pairs of bundles such that: a) one bundle in each pair

183 included a higher-value canteen voucher than the other, which in turn included a higher value

184 supermarket voucher; b) the value of each type of voucher included in each bundle was at least 4

185 (to allow for an inferior bundle to be added later - see below) but at most 18, and was a

186 multitude of 2 (consistent with the resolution of the grid, and to allow for enough distance

187 between the areas of interest). We drew the pairs of bundles randomly from this set,

188 independently for each subject and without replacement (ensuring that no choice sets were

189 duplicate within subjects). We treated the first three trials obtained in this way as training,

190 familiarizing subjects with the problem. 
191 For each of the following 27 trials (trials 4 to 30), we constructed an additional, 'matching' trial,

192 by adding a third, decoy bundle to the original two. The decoy was set randomly on each

193 occasion, but always inferior to one (and only one) of the other two bundles (target). That is, the

194 decoy was drawn from the set of bundles which: a) contained strictly less of one type of voucher

195 and no more of the other type of voucher than one of the existing two bundles (the target), but

196 strictly more of one type of voucher (and strictly less of the other type) than the competitor; b)

197 contained at least 2 but at most 16 of each type of voucher (a multitude of 2). In the example

198 shown in Figure 2, the matching trial would include a decoy located either north of bundle 2 but

199 to the south-west of bundle 1 , or east of bundle 1 and to the south-west of bundle 2 .

200 The 27 matching trials were scheduled after the initial trials, in a randomized order. The

201 reason for this relative timing of the two blocks was that, in the first instance, we wished to elicit

202 each subject's genuine preference between the competitor and the target without any current or

203 past exposure to decoys. At the same time, in the 'Results' section we report that the distractor

204 tasks were apparently successful in eliminating the influence of subjects' potential recollections

205 of the initial trials on their behavior and attention patterns in the matching trials, i.e. the ordering

206 of the blocks was unlikely to interfere with our study of choice reversals in the matching trials.

\section{Payoffs}

208 Subjects were told that, upon completion, they would receive a single voucher bundle,

209 drawn randomly from the set of the bundles they selected throughout all trials. This kind of

210 random lottery payoff mechanism is often used, as it is effective in preventing portfolio building

211 strategies and ensuring the independence of actions across trials, while allowing for 'real-world

212 consequences' of choices (Cubitt, Starmer, \& Sugden, 1998). On average, subjects received an 
213 equivalent of 7 USD in vouchers, and the whole study took 25 minutes to complete (including

214 the reading of instructions, eye-tracking calibration, and the distractor tasks).

\section{Results}

\section{Measurements and data pre-processing}

217 For each subject and each of the trials numbered 4 to 57 (i.e. skipping the training trials),

218 we identified the eye fixations that occurred between displaying the choice screen and the subject

219 submitting the choice (a fixation is a pause of eye movement on any part of the visual field,

220 representing the subject's gaze being fixed on a specific point on the screen; see the appendix for

221 details of the fixation detection procedure). We computed the total duration (in milliseconds) of

222 all fixations directed at each of the disks representing the available choices. Henceforth, by

223 'attention to option $\mathrm{X}$ ' we mean the total duration of fixations directed at the disk representing X.

224 For each pair of matched trials (the initial trial and its later matching trial including a decoy), we

225 identified the bundle that was chosen (preferred) in the initial trial - let ' $\mathrm{P}$ ' denote this bundle, and

226 let 'NP' denote the other bundle, the one that was not chosen (preferred) in the initial trial.

227 Accordingly, we calculated the value of a variable we term 'attention reversal' (between the two 228 trials), defined as follows:

$$
\log \left[\frac{\text { attention to NP in the matching trial / attention to P in the matching trial }}{\text { attention to NP in the initial trial / attention to P in the initial trial }}\right]
$$

Thus, attention reversal is positive when more attention is allocated to NP relative to $\mathrm{P}$ in

231 the matching trial compared with the initial trial, and negative if the opposite occurs (the log-

232 ratio transformation is used to normalize the comparison outcome). In other words, the greater

233 the shift of attention between the initial and matching trial - away from the option chosen in the 
234 initial trial and towards the option not chosen in that trial - the greater the attention reversal.

235 Henceforth, we will at times for brevity refer to the option not chosen in the initial trial (NP) as

236 the 'less preferred' option, and to P as the 'more preferred' option.

237 To determine the potential role of the decoy in attention reversal, for each pair of

238 matched trials we also specified the following two variables:

239 (1) 'target-preferred', a binary variable, taking a value 1 if the target option in the matching trial

240 was P, i.e. if the decoy was set inferior to the more preferred option; if this was not the case, the

241 variable took a value 0

242 (2) 'proximity-to-target', a continuous variable, equal to the difference between the Euclidean

243 distance between the decoy and the competitor and a similar distance between the decoy and the

244 target (using a log-ratio of the two distances instead does not cause a qualitative change of the

245 results; also note that the reason why the two distances are not considered as separate variables is

246 because it is impossible to change one without changing the other, and due to their strong

247 negative correlation including both in a regression model would cause multicollinearity).

248 Lastly, an alternative dependent variable that we used in a separate model was 'choice

249 reversal' (between the matching and initial trial). This was a binary variable taking a value 1 if in

250 the matching trial the subject chose the bundle she did not choose in the initial trial (otherwise, it

251 took a value 0). Such choice reversals occurred in $19 \%$ of matched trial-pairs.

\section{Checking for memory carryover effects}

253 To begin with, we wanted to check if our distractor task was successful in removing any

254 carryover memory effects, i.e. if the subjects' behavior in the matching trials was not influenced

255 by their previous exposure to the corresponding initial trials. To this end, we estimated a mixed-

256 effects binary logistic regression model in which each pair of matched trials by a given subject 
257 constituted a single observation, with random intercept and slope effects clustered by subject to

258 allow for correlated errors. The dependent variable was 'choice reversal', and the sole

259 independent variable was the number of trials separating the initial trial from its matching trial.

260 We found no significant effect between the two variables $(\beta=-0.004, \mathrm{t}=-0.522, \mathrm{p}=.602)$

261 suggesting that a reduced time difference between the two matched trials did not significantly

262 increase the likelihood that a subject's choice would be the same in both of them. In fact, the

263 same was true for the relationship between the time difference and attention reversal $(\beta=0.002$,

$264 \mathrm{t}=0.829, \mathrm{p}=.407)$.

\section{The influence of inferiority vs. similarity on attention reversal}

Following this initial check, we proceeded to the main focus of our investigation, namely

267 the effect of 'target-preferred' and 'proximity-to-target' on attention reversal. As a prelude to the

268 main analysis, Table 1 shows the average attention reversal depending on the values of the two

269 independent variables in question. In general, attention reversal tends to be positive on average,

270 possibly due to the presence of the decoy distorting the attentional bias towards the preferred

271 option in the initial trial. However, it appears that the difference between the attention transfer

272 when target-preferred $=1$, compared with target-preferred $=0$, increases with proximity-to-

273 target. This would suggest that the attention transfer to the less preferred option brought about by

274 targeting it, rather than the more preferred option, with an inferior decoy, is greater when the

275 decoy is more strongly inferior but less similar to the target (in fact, for high proximity-to-target,

276 we observe a 'repulsion effect' of the decoy instead of attraction).

277 To evaluate the statistical significance of the above effects of the way in which the decoy 278 is set, we estimated a mixed-effects linear regression model, again with each pair of matched 
279 trials constituting a single observation and random intercept and slope effects clustered by

280 subject to allow for correlated errors. The dependent variable was now 'attention reversal', and

281 the independent variables were the 'target-preferred' and 'proximity-to-target' variables

282 described above (as well as their interaction). We dropped those pairs of matched trials in which

283 either: a) the subject selected the decoy bundle in the matching trial ( $2 \%$ of trial pairs); b)

284 fixations were not recorded in at least one of the two matched trials due to a technical

285 malfunction, looking away from the screen, blinking etc. (a further $9 \%$ of trial pairs); or c) the

286 subject only looked at one of the available options in either of the matched trials, making it

287 impossible to calculate the value of attention reversal (10\% of trial pairs; clipping the log-ratio

288 values instead of dropping these trial pairs does not cause a qualitative change of the results).

289 The fixed effects estimation results (based on robust covariances) are presented in Table 2.

$290 \quad$ [Table 2 about here]

291 The fact that the model intercept is significantly positive $(\beta=0.702, \mathrm{p}<.001)$ indicates that, when

292 the decoy is inferior to the 'less preferred' option (not chosen in the initial trial) and equally

293 distant from the target and the competitor (target-preferred = proximity-to-target $=0$ ), then in the

294 matching trial subjects allocate more attention to the less preferred option (relative to the more

295 preferred one) compared with the initial trial. In other words, a decoy equally similar to both

296 options, but inferior to the less preferred one, causes a transfer of attention towards it.

297 The significantly negative coefficient estimate of 'target-preferred' $(\beta=-0.724, p<0.001)$

298 means that making the decoy inferior to the more preferred option (instead of the less preferred

299 one), but keeping its distance from the (new) target similar to the distance from the competitor

300 (low proximity-to-target), results in a greater proportion of attention being allocated to the more 
301 preferred option. In fact, when proximity-to-target $=0$ and target-preferred $=1$, the estimated

302 attention-reversal towards the less preferred option becomes (slightly) negative. This suggests

303 that, given a low proximity-to-target, attention only transfers towards the less preferred option if

304 the decoy is inferior to it rather than to the more preferred option.

305 Most importantly, the significantly negative estimate of 'proximity-to-target' $(\beta=-0.082$,

$306 \mathrm{p}=0.002$ ) indicates that, when the decoy is inferior to the less preferred option (target-preferred =

307 0), then the smaller the extent to which it is inferior (and hence the more similar / closer to the

308 target it is relative to competitor), the smaller the transfer of attention towards the (less preferred)

309 target option. Accordingly, the significantly positive estimate of the 'target-preferred*proximity-

310 to-target' interaction term $(\beta=0.135, \mathrm{p}=.006)$ indicates that the attention transfer to the less

311 preferred option brought about by targeting it, rather than the more preferred option, with an

312 inferior decoy, is greater when the decoy is more strongly inferior but less similar to the target.

313 This tendency can also be seen in Figure 4, illustrating the regression results, with the highest

314 estimated transfer of attention towards the less preferred option (left panel, warm colours)

315 obtained for decoys that are strongly inferior but not very similar/close to that option.

316 [Figure 4 about here]

\section{The influence of inferiority vs. similarity on choice reversal}

318 To verify if the decoy-induced reversals of attention between the options translated into

319 choice reversals, we estimated a separate mixed model (this time, a binary logit one), with the

320 same fixed and random effects but with 'choice reversal' as the dependent variable. The fixed

321 effects estimation results (based on robust covariances) are presented in Table 4, preceded by the

322 raw frequencies of choice reversal (depending on the two independent variables) shown in Table

3233. 
324 [Table 3 about here]

325 [Table 4 about here]

In analogy with attention reversal, it seems from Table 3 that the frequency of choice

327 reversal decreases with proximity-to-target when target-preferred $=0$ but (in almost all cases)

328 increases with proximity-to-target when target-preferred $=1$. Consequently, the difference

329 between the frequency of choice reversal when target-preferred $=1$ vs. when target-preferred $=0$

330 increases with proximity-to-target. This would suggest that the increase in the likelihood of

331 choosing the less preferred option brought about by targeting it, rather than the more preferred

332 option, with an inferior decoy, is greater when the decoy is more strongly inferior but less similar

333 to the target.

334 Similarly, the regression coefficient estimates shown in Table 4 are all significant and

335 (apart from the intercept) have the same signs as those in Table $2^{1}$. Specifically, we find that,

336 when the decoy is set inferior to the less preferred option (target-preferred $=0$ ), then the smaller

337 the extent to which it is inferior (and hence the larger 'proximity-to-target'), the smaller the

338 chance of a choice reversal $(\beta=-0.396, \mathrm{p}<.001)$. Additionally, the significantly positive estimate

339 of the 'target-preferred*proximity-to-target' interaction $(\beta=0.636, \mathrm{p}<.001)$ indicates that the

340 increase in choice reversal likelihood brought about by targeting it, rather than the more

\footnotetext{
${ }^{1}$ Note that the fact that the intercept is not significantly different from zero simply means that the probability of choice reversal when target-preferred $=$ proximity-to-target $=0$ is not significantly different from $1 / 2$. As the link function is different in each case and 'zero' has a different interpretation, this (null) result does not mean that the current relationship is not consistent with the one estimated in Table 2.
} 
341 preferred option, with an inferior decoy, is greater when the decoy is more strongly inferior but

342 less similar to the target.

Given the apparent link between the properties of the decoy on one hand and both

344 attention and choices on the other, it seems natural to consider a model in which both attention

345 reversal and choice reversal are present at the same time. For example, one might wonder

346 whether attention reversal mediates the relationship between the decoy and choice reversal

347 demonstrated in Table 4. However, apart from the technical difficulties of carrying out a

348 mediation analysis within a mixed-model with a continuous mediator and a binary outcome

349 variable, the complex, bi-directional nature of the attention-choice link we mentioned in the

350 introduction is what prevents us from doing so. In particular, it is likely that the properties of the

351 decoy affect, in the first instance, both attention to the choice options and a latent preference

352 towards them. At the same time, the emerging preference is bound to influence attention, and

353 vice versa, until a choice is finally made according to the preference at the time.

In Table 5, we present an auxiliary regression, which differs from the one in Table 4 in

355 one feature only - namely that attention-reversal is included as an independent variable. As

356 expected, this suggests a positive link between attention-reversal and preference-reversal

357 ( $\left.\beta_{\text {attention-reversal }}=1.956, \mathrm{p}<.001\right)$, while the other coefficient estimates are similar to those in Table

358 4. Although this might suggest that attention does not mediate the link between the decoy and

359 choices, one should interpret the results of the model in Table 5 with caution, due to the fact that

360 attention-reversal and choice-reversal may be co-determined by the latent preference variable

361 that is omitted from the model, and the resulting endogeneity problem could bias the regression

362 estimates. 
Despite the lack of a straightforward causal relationship between attention and choice, we

364 believe that our results so far suggest that the link between them could potentially be exploited

365 for predictive purposes. In particular, we now explore the possibility of predicting, based on

366 attention to the choice options in the matching trial, whether or not a choice reversal has taken

367 place.

\section{Detecting choice reversals based on eye-data}

Our aim here was to check if it is possible to use eye-data to detect choice reversals ex-

370 post, i.e. to determine whether or not a subject who chose the target did so because of or

371 regardless of the decoy. While not central to our hypothesis regarding the attentional mechanism

372 underlying the attraction effect, this analysis could have important practical implications. If

373 successful, it would indicate, for example, that online retailers might in the future use their

374 customers' increasingly easy to obtain eye-data (e.g. from smartphone cameras) to determine if

375 decoys that they deployed actually change the choices of individual consumers. For example,

376 suppose that a retailer wants to establish which 'types' of consumers (say, those who spent a lot

377 at its online store in the past vs. those who did not) are most susceptible to the attraction effect

378 and can be influenced by decoys. Suppose further that a similar fraction of consumers in each

379 group who were exposed to decoys have nevertheless opted for the competitor options, i.e. were

380 clearly not influenced. However, those 'low-spenders' who chose the target tended to focus their

381 attention on it while ignoring the decoy and the competitor. In contrast, the 'high-spenders' who

382 chose the target tended to look at the competitor and the decoy a lot more. Should the retailer

383 conclude that both groups were similarly influenced by the decoy, or perhaps the 'high-spenders'

384 were more strongly influenced but also liked the target options less in the first place, giving rise

385 to a similar overall proportion of target purchases? While such questions could eventually be 
answered by continued experimentation with product offerings and analysing the aggregate sales, using eye-data could potentially help to more quickly determine which individual consumers' choices were successfully altered.

With such practical applications in mind, we wished to verify if the prediction of choice reversals from attention measures can be done 'out of sample', based on a model estimated on data

391 obtained from other people (e.g. through market research). Hence, we conducted a cross-validation 392 procedure using data from those matching trials in which the target was chosen.

394 random subject intercept, 'choice reversal' as a dependent variable, and the proportional

395 attention to the competitor and target (computed as a fraction of total fixation time) being the two

396 independent variables (the proportional attention to decoy was thus redundant and not included).

397 In Table 6, we report the model estimated for the entire sample of 68 subjects. This indicates that 398 increased attention to the competitor (not chosen by the subject) is associated with an increased 399 likelihood that the competitor has been chosen in the initial trial, i.e. that a choice reversal has 400 taken place $\left(\beta_{\text {attention-to-competitor }}=8.085, \mathrm{p}=.001\right)$. However, this effect is weaker when attention 401 to the chosen target bundle is larger, which means, for a given attention-to-competitor, that 402 attention to the decoy is smaller $\left(\beta_{\text {attention-to-competitor*attention-to-target }}=-6.935, \mathrm{p}=.048\right)$. In other 403 words, the choice is more likely to have been altered by the decoy when attention to both the 404 decoy and the competitor is large, perhaps indicating a preference for the competitor that is 405 'suppressed' by the decoy.

406 Moving on to the actual cross-validation procedure, for each subject, we estimated the 407 same model as the one in Table 6, but using data from the remaining 67 subjects. In each case, 
408 we then used the model to estimate the choice reversal likelihood in the choice trials of the one 409 subject whose data we set aside.

410 Having saved the cross-validated regression scores estimated for each subject, we then 411 conducted a signal detection analysis to evaluate the overall out-of-sample predictive power. In 412 particular, for each cross-validation fold, we computed a receiver operating characteristic curve 413 (ROC) that combines all possible false positive - sensitivity pairs (points) that would obtain 414 depending on where we set the threshold value of choice reversal likelihood estimated by the 415 regression model above which a prediction of a choice reversal is made. For instance, a retailer 416 may take a 'liberal' approach and judge as effective all those decoys it used where the estimated 417 choice reversal likelihood is not extremely low. In contrast, a 'conservative' retailer might stop 418 using all decoys that do not achieve a high choice reversal likelihood. The total area under the 419 ROC ('AUC') is a measure of the overall signal detection power, and the overall potential of 420 eye-data to inform such decisions across all possible threshold values. Formally, the AUC is 421 equivalent to the probability that the likelihood of choice reversal estimated for a randomly 422 chosen trial in which choice reversal occurs is higher than for a randomly chosen trial in which it 423 does not occur, and it is also equal to the value of the corresponding Wilcoxon-Mann-Whitney 424 test statistic.

425 In our case, we found the mean AUC to be equal to 0.62, with a 95\% Confidence Interval $426[.579 ; .663]$, obtained using the method of computing confidence intervals for cross-validated 427 AUC estimates for pooled repeated measures data sets developed by LeDell, Petersen, and van 428 der Laan (2015). Thus, the mean AUC was significantly above the chance level of 0.5 (whereby 429 a choice reversal is predicted by chance in a given fixed proportion of cases). 
Although several theories (Roe et al., 2001; J. S. Trueblood et al., 2014, and others

433 outlined in the introduction) pointed to attention transfers as a key mechanism behind the

434 attraction effect, little or no process tracing evidence to support this role of attention has been

435 provided so far. Similarly, while recent work has examined other context effects in non-

436 hypothetical choices (Pinger, Ruhmer-Krell, \& Schumacher, 2016), research on the attraction

437 effect has been predominantly based on hypothetical ones.

438 The present experiment combined eye-tracking and real economic consequences of

439 choices in a spatial grid design that allowed subjects to identify and assess both similarity and

440 inferiority relationships between choice options with similar ease. The relative strength of these

441 two relationships could be quantified, manipulated, and was included in the regression models as

442 an explanatory variable. The aim was to investigate whether the failure to replicate the attraction

443 effect under pictorial rather than abstract choice visualization could be explained by the fact that

444 the abstract choice presentation makes the inferiority relationship between the decoy and target

445 salient, while the pictorial presentation emphasizes their similarity.

446 We found that attention is transferred from one's more preferred choice option to the less

447 preferred one if a decoy option is set inferior to the less preferred option (rather than the more

448 preferred one). Crucially, this transfer of attention was stronger when the decoy was less similar

449 and hence more inferior relative to the target, suggesting that inferiority rather than similarity is

450 key for the attentional spotlight effect to occur.

451 What makes this result particularly compelling is that the spatial grid presentation of

452 choices made similarity easy to perceive by virtue of it being represented by visual proximity.

453 Existing eye-tracking research suggests that objects that are close to each other are more likely to 
454 be seen as a group and tend to attract more attention (Kimchi et al., 2016). Thus, one would

455 expect a greater transfer of attention to the target if the decoy is more similar and closer to it. The

456 fact that a significant opposite tendency occurs indicates that the 'attentional spotlight' due to the

457 decoy's inferiority to the target must be strong, as we see decoys that are further away but more

458 inferior to the target result in more attention being allocated to the latter.

459 Importantly, the decoy-induced transfer of attention seems well aligned with choice

460 reversals, as we found that these are more likely if a decoy is set inferior to one's less preferred

461 option but further away from it, so that it is less similar but more inferior relative to the target. In

462 other words, the circumstances that increase the attentional transfer also make choice reversals

463 more likely.

464 It has been argued that the attraction effect might not replicate due to the heterogeneity of 465 subjects' preferences artificially reducing its aggregate measures in between-subject designs

466 (Simonson, 2014). For instance, some people might be repulsed rather than attracted by the 467 decoy, while others might be unaffected due to strong preferences (Farmer, Warren, El-Deredy, 468 \& Howes, 2016) or a clinical condition (Farmer, Baron-cohen, \& Skylark, 2017). In the words of 469 Huber et al. (2014), 'because of heterogeneity in our goals and search experiences, it may be 470 difficult to identify whether uncovering dominance altered a decision; [...] more work in eye 471 tracking and neuroscience is needed to detect the impact of idiosyncratic dominance on choice'.

472 Our use of eye-tracking combined with mixed-models addresses these issues and demands in two 473 ways. First, the fact that we obtain significant results while allowing for random intercept and 474 slope effects suggests that the overall effect of the decoy's similarity vs. inferiority to target is 475 strong relative to the between-subject variation in this respect. In other words, the overall effect 476 is significant even after accounting for subject heterogeneity. Additionally, thanks to the 
477 repeated-measures design, we are able to identify the attentional patterns accompanying choice

478 reversals at an individual subject level, and to determine the within-subject effect of similarity

479 vs. inferiority on both attention and choice. We found that these effects are considerable, ranging

480 from an attraction towards the target for low proximity to target, to 'repulsion' at the other

481 extreme (see Table 1 and Table 3, as well as Frederick et al., 2014).

482 To further underline the link between attention and choice reversal, we also wished to

483 investigate if it is possible to predict if a decoy actually induced a change in a subject's

484 preferences in a given matching trial in which a target was chosen, based solely on how the

485 subject visually examined the available options. In other words, on the basis of how attention

486 was distributed between the decoy, the target and the competitor, we would like to predict if the

487 target has been selected because of or irrespective of the decoy, i.e. if the target would also have

488 been chosen had the decoy been absent.

489 The reason why we thought that this might be possible is as follows. If the decoy was set

490 inferior to the subject's preferred option (i.e. there was no choice reversal), then it would have

491 only reinforced the subject's tendency to look at the chosen target bundle as the more attractive

492 option. In contrast, if the target was the less preferred option (i.e. a choice reversal occurred),

493 then the decoy would have artificially inflated attention to the target, but attention to the option

494 that was not chosen might still be substantial due to it being the subject's more preferred bundle.

495 Thus, we would expect attention to the option that was not chosen to have been higher in case of

496 choice reversal than in those cases when it does not occur, and this should make it possible to

497 'diagnose' choice reversals from eye-data.

498 Indeed, following a cross-validation procedure, we obtained an 'out-of-sample'

499 classification accuracy that was significantly above chance. This further points to the role of 
500 attention in choice reversal, since, given a set of options and an observed choice, the way in

501 which attention was distributed is indicative of whether the choice in question has been affected

502 by the decoy option. It is also a fact that could, in the future, be used in the evaluation of the

503 effectiveness of decoys deployed as marketing tools. Similarly, or in further research of context

504 effects.

\section{Scope and Limitations}

Despite the novelty of using eye-tracking and non-hypothetical choices to study the

507 attraction effect, we must acknowledge certain limitations in the extent to which the results

508 would generalize beyond the present experimental framework. In particular, our presentation of

509 the choice trials as a spatial grid was different from both the abstract numerical and the pictorial

510 presentation used in previous studies. We thought that the spatial grid might, from the subjects'

511 point of view, resemble a real-world retail setting in which goods are arranged on shelves with

512 similar products located close together. Nevertheless, one might argue that similarity measured

513 as the distance in the space of product attributes is not the same as one between, say, a fresh vs. a

514 spoiled apple (i.e., as in the studies with pictorial presentation). The fact that, in our setting,

515 similarity is correlated with visual proximity could potentially cause artefacts, such as attention

516 switching from decoy to target merely due to the ease of transferring gaze over a short distance,

517 or indeed result in eye fixations on the decoy being incorrectly registered as ones on a similar

518 and spatially close target. Fortunately, the effect we have found is in the opposite direction, with

519 more attention on the target registered when the decoy is further away from it. Nevertheless,

520 future research might verify if it would still persist if the choice options were presented in a more

521 conventional manner, which would have the additional advantage of facilitating comparisons

522 with previous literature. 
A separate issue is the within-subjects design, and the fact that subjects would first

524 choose without and then with a decoy being present. This was to identify their 'true' preference, 525 undistorted by any current or previous exposure to decoys. However, it may have been that the 526 initial binary choice in some way influenced the choice in the later, matching trial, despite the 527 presence of the distractor tasks.

528 Similarly, the subjects may have noticed a pattern in the matching trials, where one of the 529 options (decoy) was always inferior compared to the target, potentially generating experimenter 530 demand effects (though the chance of this happening is reduced by the fact that the choices were 531 incentivized, and whether the experimenter would demand a choice of the target or the 532 competitor could not have been apparent to subjects). These problems could be addressed in 533 future research, perhaps via more unobtrusive, indirect ways of establishing preferences. Our 534 results on detecting choice reversals from eye-data could provide some guidance as to how this 535 might be achieved.

536 Overall, our results are consistent with and shed light on the abstract vs. pictorial

537 discrepancy reported by previous studies, which may indeed be due to the abstract representation 538 of the choice options making the inferiority relationship between decoy and target salient, and 539 the pictorial one highlighting their similarity instead, thus weakening the attraction effect. On the 540 other hand, the fact that inferiority rather than similarity is crucial for the attentional spotlight to

541 be cast by the decoy on the target can be contrasted with what is postulated by existing theories

542 (Roe et al., 2001; Trueblood et al., 2014, and others), which generally assume that similarity is

543 required for inferiority of the decoy to have an effect. Because of both the novelty and

544 limitations of our experiment design discussed above, it is too early to say if the assumptions

545 about the role of attention present in existing accounts of the attraction effect should be revised. 
546 At the same time, the effect was, in the past, demonstrated in various and often unusual settings

547 (e.g. Shafir, Waite, \& Smith, 2002; J. Trueblood, Brown, Heathcote, \& Busemeyer, 2013). In

548 line with this tradition, our work can be seen as extending the set of circumstances in which

549 attraction occurs, as well as documenting the underlying attentional patterns.

\section{Conclusions}

The present study was the first one to investigate the attentional correlates of decoy-

552 induced choice reversals on an individual-trial level, and one of the very few studies of the

553 attraction effect involving non-hypothetical choices. Combining a repeated-measures design with

554 a mixed model analysis of the data allowed for a precise identification of how the attributes of

555 the decoy vis-à-vis those of the target influence the transfer of attention towards the latter and the

556 likelihood of a choice reversal.

557 In particular, we found that a decoy does indeed draw attention towards its target and

558 away from the competitor, but that this effect is reduced as the decoy becomes more similar and

559 hence less strongly inferior relative to the target. We also found that the same circumstances also 560 reduce the choice reversal likelihood.

$561 \quad$ Our results suggest that it is the inferiority, rather than similarity of the decoy to target

562 which drives the attraction effect. This could help explain the discrepancy of results in studies

563 with abstract vs. pictorial representation of the choice options, because the former makes

564 inferiority salient, while the latter tends to emphasize similarity.

565 To further underline the link between choice reversal and attention, we showed that,

566 based on how a person looked at the available options, it is possible to predict if the presence of

567 the decoy influenced the observed choice. This suggests that eye-data could be used in the

568 assessment of marketing strategies and in the further research of context effects. 


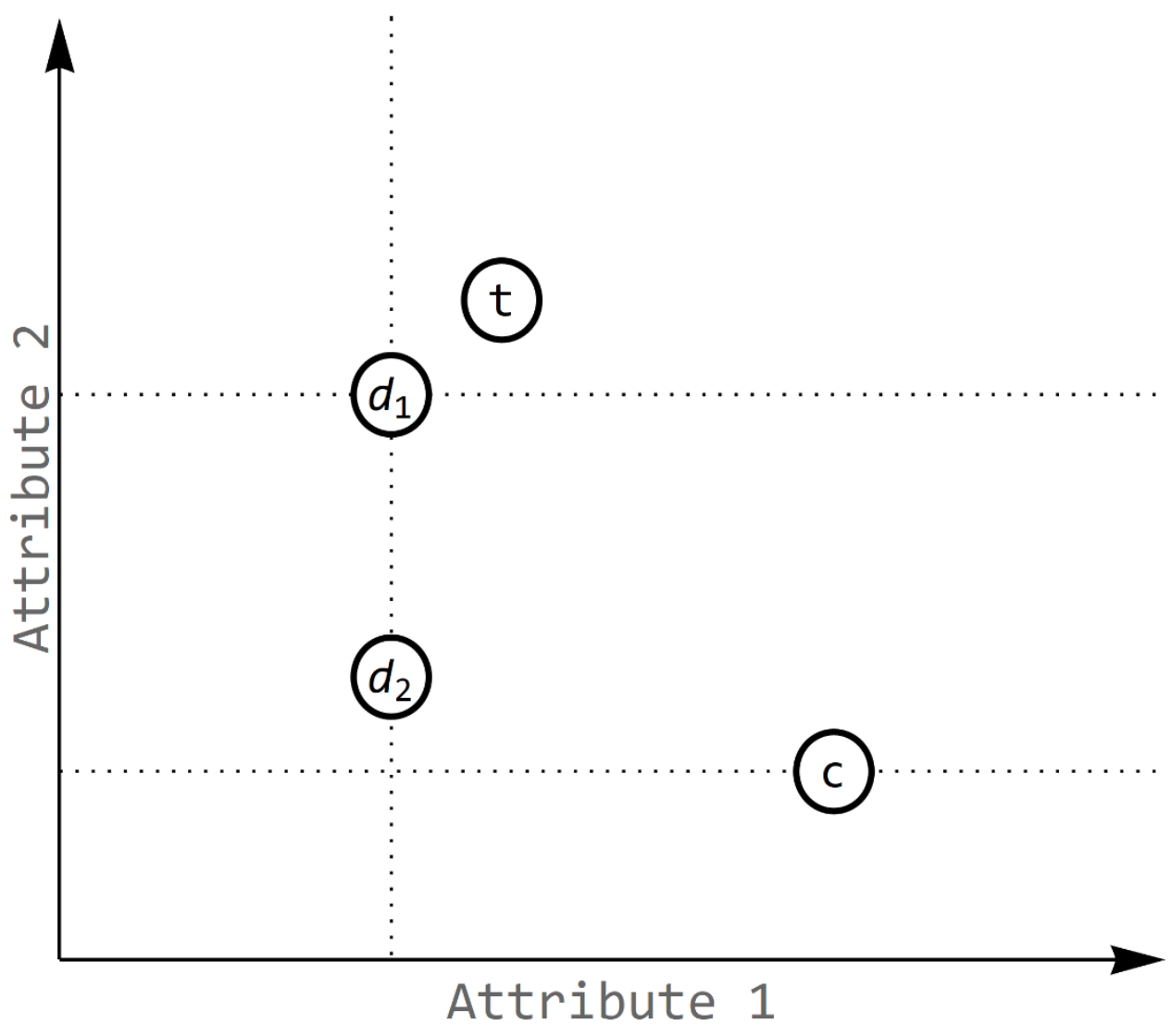

Figure 1. An example choice-problem. 


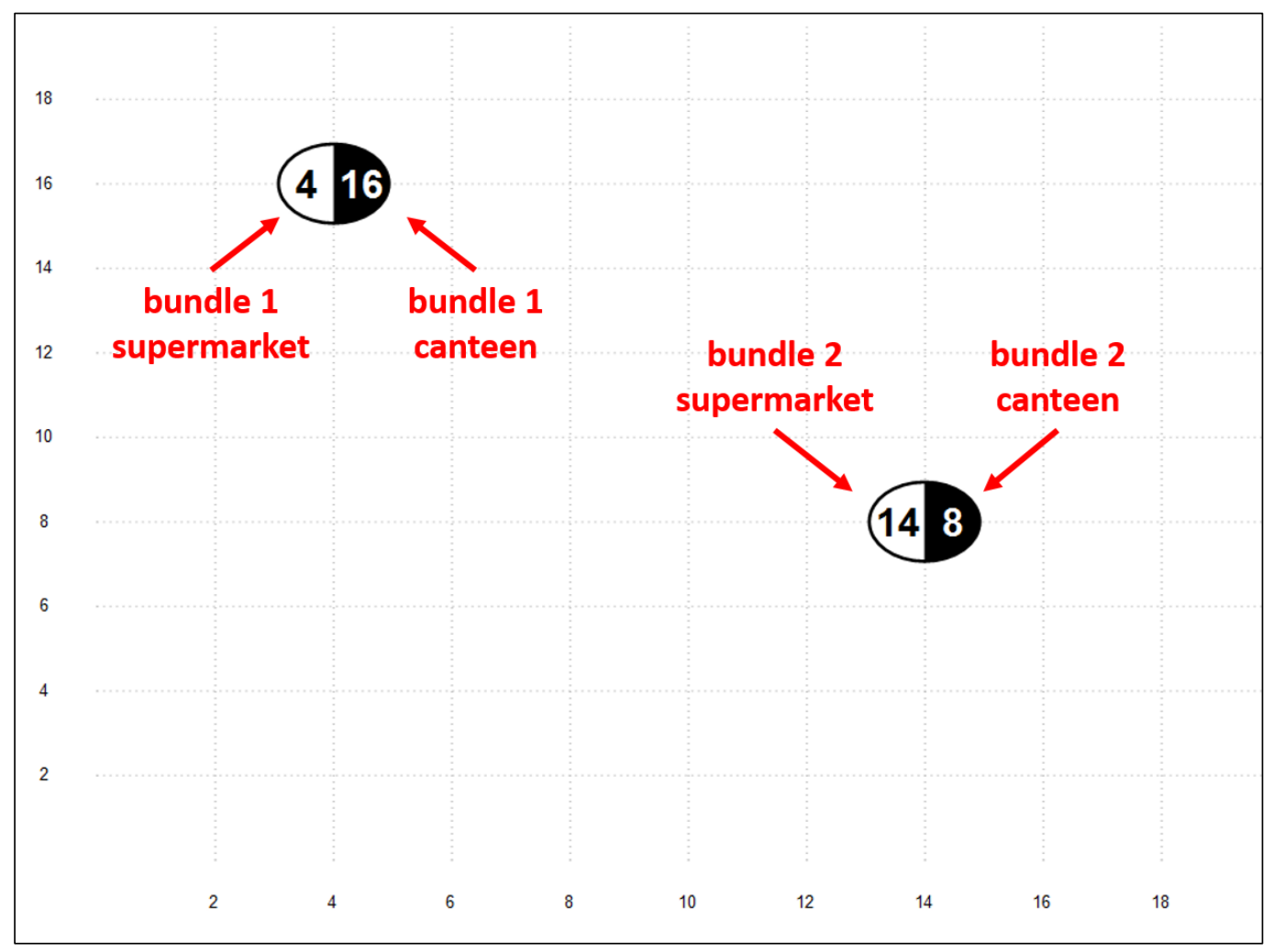

572 Figure 2. Example choice screen shown to subjects (subjects do not see the red captions); the

573 placement of canteen vs. supermarket voucher values within the disks and in the X-Y space was

574 flipped for half of the subjects.

575 


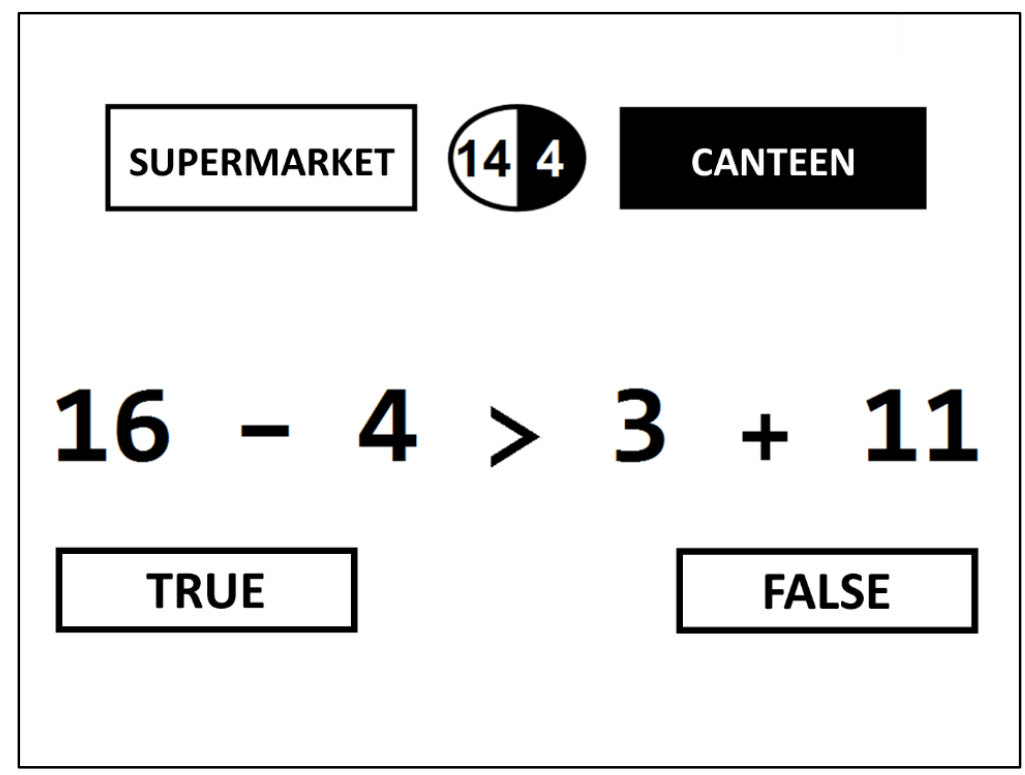

Figure 3. Example distractor task screen shown to subjects 


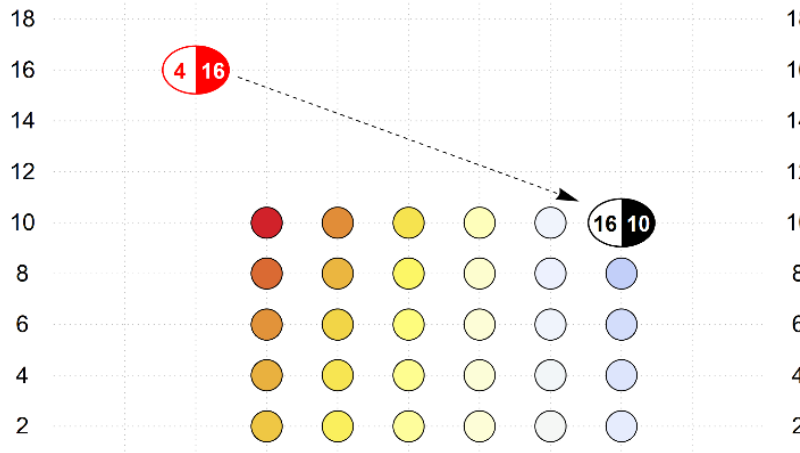

18
16
14
12
10
8
6
4
2

580 Figure 4. Illustration of the estimated regression model of attention reversal. In the example

581 shown in the left panel, the top-left of the two bundles (in red) is the 'preferred' one (chosen in

582 the initial trial). The coloured circles represent the potential locations of decoy bundles targeting

583 the 'not-preferred', bottom-right bundle (in black). The colour of each circle represents the value 584 of attention reversal, i.e. the extent to which attention is transferred towards the 'not-preferred' 585 bundle (in the direction indicated by the arrow) in the matching trial. The value of attention 586 reversal is obtained from the estimated regression for the value of proximity-to-target calculated 587 for the given decoy location and target-preferred $=0$. In the right panel, the analogous attention 588 reversal is shown in the opposite case of the bottom-right bundle having been chosen in the 589 initial trial (in this case, target-preferred $=1$ ). 
591 Table 1. Average attention reversal (across all subjects and trials) depending on the values of

592 'target-preferred' ( $1=$ decoy inferior to the option chosen in the initial trial $)$ and 'proximity-to593 target' (relative to the competitor).

\begin{tabular}{|r|r|r|}
\hline \multicolumn{3}{|c|}{ average attention reversal } \\
\hline \multirow{2}{*}{ proximity-to-target } & \multicolumn{2}{|c|}{ target-preferred } \\
\cline { 2 - 3 } & 0 & 1 \\
\hline$(-\infty, 0]$ & .792 & .142 \\
\hline$(0,2]$ & .566 & .005 \\
\hline$(2,3]$ & .508 & -.009 \\
\hline$(3,4]$ & .463 & .192 \\
\hline$(4,6]$ & .259 & .430 \\
\hline$(6, \infty)$ & .126 & .389 \\
\hline
\end{tabular}

594 
595 Table 2. Coefficient estimates of a mixed-effects model of attention reversal between the matching

596 and initial trial, modeled as a function of whether the target was set to the option preferred in the

597 initial trial and of the proximity/similarity of the decoy to the target relative to the competitor.

\begin{tabular}{|c|c|c|c|c|c|c|}
\hline \multicolumn{7}{|c|}{ attention reversal between matched trials $(\mathrm{N}=1443)$} \\
\hline \multicolumn{7}{|c|}{ fixed effects } \\
\hline & \multirow[b]{2}{*}{$\beta$} & \multirow[b]{2}{*}{ SE } & \multirow[b]{2}{*}{$\mathrm{t}$} & \multirow[b]{2}{*}{$\mathrm{p}$} & \multicolumn{2}{|c|}{$95 \% \mathrm{CI}$} \\
\hline & & & & & Lower & Upper \\
\hline intercept & 0.702 & 0.113 & 6.231 & $<.001 *$ & 0.481 & 0.924 \\
\hline target-preferred & -0.724 & 0.138 & -5.264 & $<.001 *$ & -0.994 & -0.454 \\
\hline proximity-to-target & -0.082 & 0.026 & -3.154 & $.002 *$ & -0.133 & -0.031 \\
\hline target-preferred & 0.135 & 0.048 & 2.790 & $.006^{*}$ & 0.040 & 0.230 \\
\hline & & & & \multicolumn{3}{|c|}{$(*$ significant at $\mathrm{p}<.05)$} \\
\hline \multicolumn{7}{|c|}{ random effects } \\
\hline \multicolumn{2}{|r|}{ variance } & \multicolumn{2}{|c|}{ correlation m. } & & & \\
\hline intercept & 0.294 & & & & & \\
\hline target-preferred & 0.342 & -0.98 & & & & \\
\hline proximity-to-target & 0.008 & -0.99 & 0.98 & & & \\
\hline $\begin{array}{l}\text { target-preferred } \\
\text { * proximity-to-target }\end{array}$ & 0.056 & 0.84 & -0.94 & -0.84 & & \\
\hline
\end{tabular}


599 Table 3. The frequency of choice reversal (across all subjects and trials) depending on the values 600 of 'target-preferred' and 'proximity-to-target'.

\begin{tabular}{|r|r|r|}
\hline \multicolumn{3}{|c|}{ frequency of choice reversal } \\
\hline \multirow{2}{*}{ proximity-to-target } & \multicolumn{2}{|c|}{ target-preferred } \\
\cline { 2 - 3 } & 0 & 1 \\
\hline$(-\infty, 0]$ & .385 & .100 \\
\hline$(0,2]$ & .305 & .128 \\
\hline$(2,3]$ & .209 & .160 \\
\hline$(3,4]$ & .159 & .227 \\
\hline$(4,6]$ & .128 & .406 \\
\hline$(6, \infty)$ & .100 & .214 \\
\hline
\end{tabular}

601 
602 Table 4. Coefficient estimates of a mixed-effects model of the likelihood of choice reversal

603 between the matching and initial trial, modeled as a function of whether the target was set to the

604 option preferred in the initial trial and of the proximity/similarity of the decoy to the target

605 relative to the competitor.

\begin{tabular}{|c|c|c|c|c|c|c|}
\hline \multicolumn{7}{|c|}{ choice reversal between matched trials $(\mathrm{N}=1443)$} \\
\hline & \multirow[b]{2}{*}{$\beta$} & \multirow[b]{2}{*}{ SE } & \multirow[b]{2}{*}{$\mathrm{t}$} & \multirow[b]{2}{*}{$\mathrm{p}$} & \multicolumn{2}{|c|}{$95 \% \mathrm{CI}$} \\
\hline & & & & & Lower & Upper \\
\hline intercept & -0.369 & 0.241 & -1.535 & .125 & -0.841 & 0.103 \\
\hline target-preferred & -1.801 & 0.305 & -5.897 & $<.001 *$ & -2.400 & -1.202 \\
\hline proximity-to-target & -0.396 & 0.067 & -5.888 & $<.001 *$ & -0.529 & -0.264 \\
\hline target-preferred & 0.636 & 0.100 & 6.345 & $<.001 *$ & 0.440 & 0.833 \\
\hline \multicolumn{7}{|c|}{ random effects } \\
\hline \multicolumn{2}{|r|}{ variance } & \multicolumn{2}{|c|}{ correlation m. } & & & \\
\hline intercept & 1.277 & & & & & \\
\hline target-preferred & 0.502 & -0.96 & & & & \\
\hline proximity-to-target & 0.058 & -0.50 & 0.71 & & & \\
\hline $\begin{array}{c}\text { target-preferred } \\
* \text { proximity-to-target }\end{array}$ & 0.108 & 0.88 & -0.98 & -0.85 & & \\
\hline
\end{tabular}

606 
607 Table 5. Coefficient estimates of a mixed-effects model of the likelihood of choice reversal

608 between the matching and initial trial, based on the same specification as the one in Table 4, but 609 with attention-reversal added as an independent variable.

\begin{tabular}{|c|c|c|c|c|c|c|}
\hline \multicolumn{7}{|c|}{ choice reversal between matched trials } \\
\hline & \multirow[b]{2}{*}{$\beta$} & \multirow[b]{2}{*}{$\mathrm{SE}$} & \multirow[b]{2}{*}{$\mathrm{t}$} & \multirow[b]{2}{*}{$\mathrm{p}$} & \multicolumn{2}{|c|}{$95 \% \mathrm{CI}$} \\
\hline & & & & & Lower & Upper \\
\hline intercept & -1.932 & 0.303 & -6.366 & $<.001 *$ & -2.527 & -1.337 \\
\hline target-preferred & -1.393 & 0.442 & -3.154 & $.002 *$ & -2.260 & -0.527 \\
\hline proximity-to-target & -0.447 & 0.094 & -4.777 & $<.001 *$ & -0.630 & -0.263 \\
\hline $\begin{array}{r}\text { target-preferred } \\
* \text { proximity-to-target }\end{array}$ & 0.688 & 0.148 & 4.660 & $<.001 *$ & 0.398 & 0.977 \\
\hline \multirow[t]{2}{*}{ attention-reversal } & 1.956 & 0.132 & 14.819 & $<.001 *$ & 1.697 & 2.215 \\
\hline & & & & \multicolumn{3}{|c|}{$\left({ }^{*}\right.$ significant at $\left.\mathrm{p}<.05\right)$} \\
\hline \multicolumn{7}{|c|}{ random effects } \\
\hline \multicolumn{2}{|r|}{ variance } & \multicolumn{2}{|c|}{ correlation m. } & & & \\
\hline intercept & 3.035 & & & & & \\
\hline target-preferred & 1.339 & -0.87 & & & & \\
\hline proximity-to-target & 0.095 & -0.46 & 0.54 & & & \\
\hline attention-reversal & 0.186 & -0.21 & -0.28 & -0.30 & & \\
\hline $\begin{array}{l}\text { target-preferred } \\
\text { *proximity-to-target }\end{array}$ & 0.242 & 0.81 & -0.87 & -0.87 & 23 & \\
\hline
\end{tabular}


610 Table 6. Coefficient estimates of a mixed-effects logistic regression model of the likelihood of

611 choice reversal between the matching and initial trial, for matching trials in which the target was

612 chosen, modeled as a function of proportional attention (fixation time) to the competitor and the

613 target in the matching trial.

\begin{tabular}{|c|c|c|c|c|c|c|}
\hline \multicolumn{7}{|c|}{ choice reversal between matched trials } \\
\hline & \multirow[b]{2}{*}{$\beta$} & \multirow[b]{2}{*}{$\mathrm{SE}$} & \multirow[b]{2}{*}{$\mathrm{t}$} & \multirow[b]{2}{*}{$\mathrm{p}$} & \multicolumn{2}{|c|}{$95 \% \mathrm{CI}$} \\
\hline & & & & & Lower & Upper \\
\hline intercept & -2.792 & 1.102 & -2.533 & $.012 *$ & -4.955 & -0.628 \\
\hline attention-to-competitor & 8.085 & 2.338 & 3.458 & $.001 *$ & 3.495 & 12.674 \\
\hline attention-to-target & 1.339 & 1.315 & 1.019 & .309 & -1.242 & 3.920 \\
\hline $\begin{array}{l}\text { attention-to-competitor } \\
* \text { attention-to-target }\end{array}$ & -6.935 & 3.530 & -1.964 & $.048 *$ & -13.869 & -0.005 \\
\hline & & & & & nificant a & $p<.05)$ \\
\hline
\end{tabular}

614

615 
617 The stimulus presentation software was programmed in Wolfram Mathematica. Each subject was

618 seated at a computer terminal, operating Microsoft Windows 8, with a 15.4-inch screen with

619 resolution set to $1280 \times 720$, and a SensoMotoric Instruments RED250 mobile eye-tracking device

620 attached underneath the screen and set to $250 \mathrm{~Hz}$ frequency. Prior to the commencement of the

621 study, we conducted a standard five-point semi-automatic calibration and validation procedure

622 (the average deviation was below $0.5^{\circ}$ for all subjects). Each trial was preceded by a fixation

623 cross, and subjects chose their preferred choice option using keyboard or mouse. A headrest was

624 used to stabilize the head position and ensure that the distance between the subject's eyes and the

625 device was approximately $65 \mathrm{~cm}$. We used the SMI Vision high-speed fixation detection

626 algorithm with standard settings (minimum fixation duration $=50 \mathrm{~ms}$, peak saccade velocity

627 threshold 40 \%s). All studies were approved by the local Faculty Research Ethics Committee. 
631 Ahn, S., Kim, J., \& Ha, Y.-W. (2015). Feedback weakens the attraction effect in repeated 632 choices. Marketing Letters, 26(4), 449-459. https://doi.org/10.1007/s11002-014-9281-6

633 Ariely, D., \& Wallsten, T. S. (1995). Seeking Subjective Dominance in Multidimensional Space:

634 An Explanation of the Asymmetric Dominance Effect. Organizational Behavior and Human Decision Processes, 63(3), 223-232. https://doi.org/10.1006/obhd.1995.1075

Ashby, N. J. S., Jekel, M., Dickert, S., \& Glöckner, A. (2016). Finding the right fit: A comparison of process assumptions underlying popular drift-diffusion models. Journal of Experimental Psychology: Learning, Memory, and Cognition, 42(12), 1982.

Bhatia, S. (2013). Associations and the accumulation of preference. Psychological Review,

Bjork, R. A., \& Whitten, W. B. (1974). Recency-sensitive retrieval processes in long-term free recall. Cognitive Psychology, 6(2), 173-189.

643 Bordalo, P., Gennaioli, N., \& Shleifer, A. (2013). Salience and Consumer Choice. Journal of Political Economy, 121(5), 803-843. https://doi.org/10.1086/673885

645 Cubitt, R. P., Starmer, C., \& Sugden, R. (1998). On the Validity of the Random Lottery Incentive System. Experimental Economics, 1, 115-131.

647 Farmer, G. D., Baron-cohen, S., \& Skylark, W. J. (2017). People With Autism Spectrum Conditions Make More Consistent Decisions. https://doi.org/10.1177/0956797617694867

649 Farmer, G. D., Warren, P. A., El-Deredy, W., \& Howes, A. (2016). The Effect of Expected 650 Value on Attraction Effect Preference Reversals. Journal of Behavioral Decision Making. https://doi.org/10.1002/bdm.2001

652 Frederick, S., Lee, L., \& Baskin, E. (2014). The Limits of Attraction. Journal of Marketing 
Research, 51(4), 487-507. https://doi.org/10.1509/jmr.12.0061

654 Hedgcock, W. M., Rao, R. S., \& Chen, H. A. (2016). Choosing to Choose: The Effects of Decoys and Prior Choice on Deferral. Management Science, 1909, 1-25.

Hedgcock, W., Rao, A. R., \& Chen, H. (Allan). (2009). Could Ralph Nader's Entrance and Exit Have Helped Al Gore? The Impact of Decoy Dynamics on Consumer Choice. Journal of Marketing Research, 46(June), 330-343. https://doi.org/10.1509/jmkr.46.3.330

Hu, J., \& Yu, R. (2014). The neural correlates of the decoy effect in decisions. Frontiers in Behavioral Neuroscience, 8, 271. https://doi.org/10.3389/fnbeh.2014.00271

Huber, J., Payne, J. W., \& Puto, C. (1982). Adding Asymmetrically Dominated Alternatives: Violations of Regularity and the Similarity Hypothesis. Journal of Consumer Research, 9(1), 90. https://doi.org/10.1086/208899

Huber, J., Payne, J. W., \& Puto, C. P. (2014). Let's Be Honest About the Attraction Effect. Journal of Marketing Research, 51(4), 520-525. https://doi.org/10.1509/jmr.14.0208

Hutchinson, J. W., Kamakura, W. A., \& Lynch, J. G. (2000). Unobserved Heterogeneity as an Alternative Explanation for "Reversal" Effects in Behavioral Research. Journal of Consumer Research, 27(3), 324-344. https://doi.org/10.1086/317588

Kimchi, R., Yeshurun, Y., Spehar, B., \& Pirkner, Y. (2016). Perceptual organization, visual attention, and objecthood. Vision Research, 126, 34-51. https://doi.org/https://doi.org/10.1016/j.visres.2015.07.008

Krajbich, I., Armel, C., \& Rangel, A. (2010). Visual fixations and the computation and comparison of value in simple choice. Nature Neuroscience, 13(10), 1292-8. https://doi.org/10.1038/nn.2635 
Król, M., \& Król, M. E. (in press). Learning from Peers’ Eye Movements in the Absence of Expert Guidance: a Proof of Concept Using Laboratory Stock Trading, Eye Tracking, and Machine Learning. Cognitive Science.

LeDell, E., Petersen, M., \& van der Laan, M. (2015). Computationally efficient confidence intervals for cross-validated area under the ROC curve estimates. Electronic Journal of Statistics, 9(1), 1583-1607. https://doi.org/10.1214/15-EJS1035

Li, V., Michael, E., Balaguer, J., Herce Castañón, S., \& Summerfield, C. (2018). Gain control explains the effect of distraction in human perceptual, cognitive, and economic decision making. Proceedings of the National Academy of Sciences, 115(38), E8825--E8834. https://doi.org/10.1073/pnas.1805224115

Lichters, M., Bengart, P., Sarstedt, M., \& Vogt, B. (2017). What really matters in attraction effect research: when choices have economic consequences. Marketing Letters, 28(1), 127138. https://doi.org/10.1007/s11002-015-9394-6

Lichters, M., Sarstedt, M., \& Vogt, B. (2015). On the practical relevance of the attraction effect: A cautionary note and guidelines for context effect experiments. AMS Review, 5(1-2), 1-19. https://doi.org/10.1007/s13162-015-0066-8

Lleras, J. S., Masatlioglu, Y., Nakajima, D., \& Ozbay, E. Y. (2017). When more is less: Limited consideration. Journal of Economic Theory, 170, 70-85. https://doi.org/10.1016/j.jet.2017.04.004

Malkoc, S. A., Hedgcock, W., \& Hoeffler, S. (2013). Between a rock and a hard place: The failure of the attraction effect among unattractive alternatives. Journal of Consumer Psychology, 23(3), 317-329.

Meier, B. P., \& Robinson, M. D. (2004). Why the Sunny Side Is Up: Associations Between 
Affect and Vertical Position. Psychological Science, 15(4), 243-247. https://doi.org/10.1111/j.0956-7976.2004.00659.x

701

702

703

704

705

706

707

708

Mogilner, C., Rudnick, T., \& Iyengar, S. S. (2008). The Mere Categorization Effect: How the Presence of Categories Increases Choosers' Perceptions of Assortment Variety and Outcome Satisfaction. Journal of Consumer Research, 35(2), 202-215. https://doi.org/10.1086/588698

Mohr, P. N. C., Heekeren, H. R., \& Rieskamp, J. (2017). Attraction Effect in Risky Choice Can Be Explained by Subjective Distance between Choice Alternatives. Scientific Reports, 7(1). https://doi.org/10.1038/s41598-017-06968-5

Ok, E. A., Ortoleva, P., \& Riella, G. (2015). Revealed ( P ) Reference Theory. American Economic Review, 105(1), 299-321. https://doi.org/10.1161/ATVBAHA.107.161042

Pinger, P., Ruhmer-Krell, I., \& Schumacher, H. (2016). The compromise effect in action: Lessons from a restaurant's menu. Journal of Economic Behavior \& Organization, 128, 1434. https://doi.org/https://doi.org/10.1016/j.jebo.2016.04.017

Reutskaja, E., Nagel, R., Camerer, C. F., \& Rangel, A. (2011). Search Dynamics in Consumer Choice under Time Pressure: An Eye- Tracking Study. The American Economic Review, 101(2), 900-926. https://doi.org/10.1257/aer.101.2.900

Roe, R. M., Busemeyer, J. R., \& Townsend, J. T. (2001). Multialternative decision field theory: A dynamic connectionst model of decision making. Psychological Review, 108(2), 370.

Samek, A., Hur, I., Kim, S.-H., \& Yi, J. S. (2016). An experimental study of the decision process with interactive technology. Journal of Economic Behavior \& Organization, 130, 20-32. https://doi.org/https://doi.org/10.1016/j.jebo.2016.06.004

Shafir, S., Waite, T., \& Smith, B. (2002). Context-dependent violations of rational choice in 

honeybees ( Apis mellifera ) and gray jays ( Perisoreus canadensis ). Behavioral Ecology and Sociobiology, 51(2), 180-187. https://doi.org/10.1007/s00265-001-0420-8

724 Shimojo, S., Simion, C., Shimojo, E., \& Scheier, C. (2003). Gaze bias both reflects and

725 influences preference. Nature Neuroscience, 6(12), 1317-1322. https://doi.org/10.1038/nn1150

727 Simonson, I. (2014). Vices and Virtues of Misguided Replications: The Case of Asymmetric Dominance. Journal of Marketing Research, 51(4), 514-519.

Smith, S. M., Roster, C. A., Golden, L. L., \& Albaum, G. S. (2016). A multi-group analysis of https://doi.org/10.1509/jmr.14.0093 online survey respondent data quality: Comparing a regular USA consumer panel to MTurk samples. Journal of Business Research, 69(8), 3139-3148. https://doi.org/https://doi.org/10.1016/j.jbusres.2015.12.002

Trueblood, J., Brown, S., Heathcote, A., \& Busemeyer, J. (2013). Not Just for Consumers: Context Effects Are Fundamental to Decision Making. Psychological Science, 24(6), 901908. https://doi.org/10.1177/0956797612464241

Trueblood, J. S., Brown, S. D., \& Heathcote, A. (2014). The Multiattribute Linear Ballistic Accumulator Model of Context Effects in Multialternative Choice. https://doi.org/10.1037/a0036137

Yang, S., \& Lynn, M. (2014). More Evidence Challenging the Robustness and Usefulness of the 